\title{
Identification of lymphatic pathway involved in the spreading of prostate cancer by fluorescence navigation approach with intraoperatively injected indocyanine green
}

\author{
Shogo Inoue, MD; ${ }_{i}^{* t}$ Hiroaki Shiina, MD; Naoko Arichi, MD; ${ }^{*}$ Yozo Mitsui, MD; ; Takeo Hiraoka, MD; \\ Koji Wake, MD; Masahiro Sumura, MD; ; $^{*}$ Satoshi Honda, MD;* Hiroaki Yasumoto, MD; ${ }^{*}$ Shinji Urakami, MD;* \\ Akio Matsubara, MD; Mikio Igawa, MD*
}

*Department of Urology, Shimane University School of Medicine, Izumo, Japan; ‘Department of Urology, Graduate School of Biomedical Sciences, Hiroshima University, Hiroshima, Japan

Cite as: Can Urol Assoc J 2011;5(4):254-9; D01:10.5489/cuaj.10159

See related article on page 260 .

\section{Abstract}

Objective: The objective of this study was to identify lymphatic vessels draining from the prostate by using a fluorescence navigation (FN) system.

Methods: Fourteen subjects were candidates for radical retropubic prostatectomy (RRP) and pelvic lymph node dissection (PLND). After an indocyanine green solution was injected into the prostate during RRP, lymphatic vessels draining from the prostate were analyzed using a FN system. After PLND based on lymphatic mapping by the FN system (in vivo probing) was performed in the external iliac, obturator and internal iliac regions; the fluorescence of the removed lymph nodes (LNs) was analyzed on the bench (ex vivo probing).

Results: Under in vivo and ex vivo probing, the fluorescence intensity of internal iliac nodes was greater than that of external iliac or obturator nodes.

Conclusion: The current study suggests that using a FN system after injecting indocyanine green is a safe and rational approach for detecting the lymphatic channel draining from the prostate. The major lymphatic pathway involved in the spreading of prostate cancer appears to relate to internal iliac LNs, which would mean that the standard PLND covering external iliac and obturator regions would not keep the cancer from spreading.

\section{Résumé}

Objectif : L'objectif de l'étude était de repérer les vaisseaux lymphatiques quittant la prostate à l'aide d'un système d'imagerie par fluorescence (IF).

Méthodologie : Quatorze sujets devaient subir une prostatectomie radicale rétropubienne (PRR) et une lymphadénectomie pelvienne. Après injection d'une solution de vert d'indocyanine dans la prostate pendant la PRR, les vaisseaux lymphatiques drainant la prostate ont été analysés par IF. Une lymphadénectomie pelvienne fondée sur la cartographie lymphatique par IF (exploration in vivo) a ensuite été réalisée dans les régions de la fosse iliaque externe, de l'obturateur et de la fosse iliaque interne; la fluorescence des ganglions lymphatiques retirés a été analysée sans délai (exploration ex vivo).

Résultats : Lors de l'exploration in vivo et ex vivo, l'intensité de la fluorescence des ganglions iliaques internes était plus forte que celle des ganglions iliaques externes ou des ganglions obturateurs. Conclusion : Cette étude porte à croire que I'IF après injection de vert d'indocyanine est une méthode sûre et rationnelle pour repérer les vaisseaux lymphatiques drainant la prostate. La principale voie lymphatique de propagation du cancer de la prostate semble être reliée aux ganglions lymphatiques iliaques internes, ce qui signifie que la lymphadénectomie pelvienne standard retirant les ganglions iliaques externes et obturateurs n'empêcherait pas le cancer de se propager.

\section{Introduction}

The concept of the sentinel lymph node (SLN) is defined as the first lymph node (LN) which is drained from the primary tumour; secondary cancer cells are not found in LNs downstream from the first one. The SLN concept has been well-established in breast and skin cancers, ${ }^{1,2}$ and was first applied to penile cancer in 1977 by Cabanas. ${ }^{3}$ In 1999, Wawroschek and colleagues first reported the possibility of applying the SLN concept to prostate cancer by using $\gamma$-probe-guided SLN dissection, ${ }^{4}$ but their approach combining preoperative lymphoscintigraphy with intraoperative $\gamma$ probing was complicated and difficult to use. Now, there are two approaches to identify SLNs. The first is the dye-guided approach, which appears to be convenient and safe, but sometimes cannot precisely detect SLNs deep in the pelvis, because the contrast of the target tissue is insufficient. The second is the radioisotope-guided (RI-guided) approach, 
Lymphatic pathway identified by fluorescence navigation

which can accurately determine the location of even deep SLNs, but cannot identify minute lymphatic vessels. ${ }^{5}$

To identify the SLN in prostate cancer, one must understand the anatomy of the lymphatic network and its flow around the prostate. Normal prostate lymphatics drain into the periprostatic subcapsular network, from which originate three groups of pathways: (1) the ascending principle pathway running along inferior vesical vessels to internal iliac $\mathrm{LNs}$; (2) the second lateral pathway going over bladder to external iliac LNs, forming a perivesical plexus; and (3) the posterior pathway running along the lateral side of the rectum reaching promontorial and lateral sacral LNs. ${ }^{6}$ Although the pathway by which prostate cancer cells leave the prostate capsule through the lymphatics and perineural spaces remains to be elucidated, the conventional and standard pelvic LN dissection (PLND) procedure has focused on the areas covering the external and obturator nodes, but not on the internal iliac nodes.

We have, therefore, developed a novel method of SLN identification assisted by indocyanine green (ICG) fluorescence during RRP. Sentinel lymph node biopsy under ICG fluorescence guidance is considered safe and useful in breast cancer. ${ }^{7}$ However, to our knowledge, there have been no reports in which ICG fluorescence navigation (FN) was used for SLN mapping in prostate cancer patients. The purpose of this study was to demonstrate a safe and convenient approach to identifying lymphatic vessels draining from the prostate and to validate the SLN concept in prostate cancer.

\section{Materials and methods}

\section{Patients}

There were 14 subjects in this study; they underwent systemic 8-core biopsy and were candidates for RRP and PLND. The surgery for RRP with PLND was performed between May 2007 and April 2008 at the Shimane University Hospital, Japan. Informed consent to participate in this study was obtained from all patients in a written form after giving sufficient explanation to the patients and their families according to the documentation approved by our institutional ethics committee. The median age at diagnosis was 68 years (range: 63-75 years) and the median prostate-specific antigen (PSA) level at diagnosis was $12.16 \mathrm{ng} / \mathrm{mL}$ (range: 4.68$52.67 \mathrm{ng} / \mathrm{mL}$ ). The median estimated prostate volume was $32.2 \mathrm{~g}$ (range: 16.8-104 g). The median number of cores positve was 2 (range: 1-8) and the median Gleason score was 8 (range: 6-9) (Table 1).

\section{ICG injection and identification of lymphatic channels}

Within 5 minutes after the $0.5 \mathrm{~mL}$ solution with $0.25 \%$ ICG (Diagnogreen 0.25\%; Daiichi Pharmaceutical, Tokyo, Japan)

\begin{tabular}{cccccc}
\hline \multicolumn{6}{l}{ Table 1. Patients' characteristics } \\
\hline Case & Age & PSA & $\begin{array}{c}\text { Prostate } \\
\text { volume }\end{array}$ & $\begin{array}{c}\text { No. cores } \\
\text { positive }\end{array}$ & $\begin{array}{c}\text { Gleason } \\
\text { score }\end{array}$ \\
\hline 1 & 65 & 12.11 & 31.6 & 2 & 7 \\
2 & 67 & 15.48 & 75.9 & 2 & 8 \\
3 & 61 & 14.04 & 32.8 & 1 & 8 \\
4 & 71 & 7.86 & 51.0 & 1 & 6 \\
5 & 65 & 18.70 & 47.1 & 1 & 7 \\
6 & 66 & 52.67 & 17.7 & 6 & 9 \\
7 & 68 & 25.12 & 30.6 & 6 & 9 \\
8 & 75 & 4.68 & 43.6 & 8 & 9 \\
9 & 64 & 8.32 & 24.8 & 7 & 7 \\
10 & 73 & 24.06 & 17.0 & 2 & 8 \\
11 & 68 & 4.74 & 16.8 & 3 & 8 \\
12 & 75 & 12.21 & 26.8 & 1 & 8 \\
13 & 70 & 6.30 & 104 & 1 & 6 \\
14 & 63 & 10.68 & 34.8 & 1 & 7 \\
\hline PSA & 6 & & & & \\
\hline
\end{tabular}

PSA: prostate-specific antigen.

was injected into each lobe of the prostate during RRP, lymphatic vessels draining from the prostate were visualized by the FN system and the timing of ICG detection time was not dependent. Indocyanine green absorbs light in the nearinfrared range and has its peak absorption at a wavelength of $800 \mathrm{~nm}$, and it emits with maximal fluorescence at a wavelength of $840 \mathrm{~nm}$, when bound to plasma proteins. The fluorescence wavelength longer than the excitation one is specific to ICG. Autofluorescence of the pelvic tissue and blood is relatively low (in the $800-\mathrm{nm}$ wavelength range), making the signal-to-noise ratio high in ICG fluorescence imaging with the PDE infrared camera system (Hamamatsu Photonics, Hamamatsu, Japan). The light source in this system contains 36 light-emitting diodes producing light with a wavelength of $760 \mathrm{~nm}$, and the detector is a charge-coupleddevice camera equipped with a filtre eliminating light with wavelengths below $820 \mathrm{~nm}$. The excitation light penetrates deeply into the pelvic tissues and the strong fluorescence of ICG enables even small particles of ICG bound to plasma proteins in the lymphatic vessels and LNs to be detected. The fluorescence signals are transmitted to a digital video processor and displayed on a TV monitor in a real-time mode.

\section{PLND}

The LNs in three regions (external iliac, obturator fossa and internal iliac regions) were carefully dissected out coupled with the surrounding adipose tissue. If a fluorescent spot kept shining after the initial LN dissection, residual LNs were dissected as well. On the basis of lymphatic mapping under FN guidance (in vivo probing), PLND was performed focusing on the external iliac, obturator and internal iliac regions. Subsequently, the fluorescence of the removed LNs was analyzed on the bench (ex vivo probing). The LN involvement was confirmed by Hematoxylin and Eosin staining of 
Inoue et al.

formalin-fixed samples and all LNs were examined pathologically. After conversion to the static image, the fluorescence intensity of LNs detected by the FN system was calculated as a relative ratio by using Imagej software.

\section{Statistical analysis}

All values were expressed as means and the results were analyzed using ANOVA followed by post-hoc test or unpaired t-test. A $p$ value of less than 0.05 was considered statistically significant.

\section{Results}

We summarized the postoperative pathology (Table 2). There were no patients with complications or adverse events associated with the intraprostatic ICG injection. Lymphatic pathways along inferior vesical vessels to internal iliac LNs were clearly illustrated in 12 cases regardless of estimated prostate volume, whereas the detection of the other channels, such as pathways to external iliac LNs, was not prominent in this study.

In case \#5, the pathological examination did not show any LN metastasis and the in vivo probing with the FN system showed highly fluorescent lymphatic flow in the internal iliac region after direct injection of ICG. The lymphatic stream having ICG particles was identified as a shining stream or spot in the fluorescence image (Fig. 1, parts A and B). Rapid venous flow with immediate loss of fluorescence could be distinguished from the lymphatic stream. The highly fluorescent region was not evident after LN dissection. The FN system did not show residual LNs, and their absence was confirmed by the naked-eye examination (Fig. 1 , parts $C$ and D). Lymph nodes stained with ICG were not evident to the naked eye. In ex vivo probing with the FN system, the fluorescence of the internal iliac LN was greater than that of the external iliac or obturator LN (Fig. 1, parts $\mathrm{E}$ and $\mathrm{F}$ ). Fluorescence of the LN was clearly detected for at least 2 hours. In case \#8 and \#9, pathological examination showed LN metastasis and there was no strong fluorescence in the regional LNs of the prostate either by in vivo or ex vivo probing.

In case \#8, the value of serum PSA was $4.68 \mathrm{ng} / \mathrm{mL}$. Though pathological examination showed $\mathrm{LN}$ metastasis in the left obturator $\mathrm{LN}$, there was no strong fluorescence in the regional LNs of the prostate either in vivo or ex vivo. On the other hand, there was a highly fluorescent region in the perivesical plexus, which was outside the extended LN dissection (Fig. 2, part A). The Gleason score from the surgical specimen was $4+5=9$. Pathological examination of the prostate revealed adenocarcinoma in most of the prostate; this expanded beyond the prostatic capsule and diffusely invaded lymphatic vessels. Pathological examination

\begin{tabular}{|c|c|c|c|c|c|c|}
\hline \multirow{2}{*}{ Case } & \multirow{2}{*}{$\begin{array}{c}\text { Patho- } \\
\text { logical T } \\
\text { stage }\end{array}$} & \multirow{2}{*}{$\begin{array}{c}\text { Gleason } \\
\text { score }\end{array}$} & \multirow{2}{*}{ Side } & \multicolumn{3}{|c|}{ Lymph node (mets) } \\
\hline & & & & External & Obturator & Interna \\
\hline \multirow[t]{2}{*}{1} & $\mathrm{~T} 2 \mathrm{~b}$ & 7 & $\mathrm{R}$ & 1 & 2 & 0 \\
\hline & & & L & 1 & 3 & 3 \\
\hline \multirow[t]{2}{*}{2} & Т3a & 8 & $\mathrm{R}$ & 0 & 1 & 0 \\
\hline & & & L & 1 & 1 & 1 \\
\hline \multirow[t]{2}{*}{3} & T2a & 7 & $\mathrm{R}$ & 3 & 2 & 1 \\
\hline & & & L & 3 & 1 & 1 \\
\hline \multirow[t]{2}{*}{4} & $\mathrm{~T} 2 \mathrm{~b}$ & 7 & $\mathrm{R}$ & 2 & 2 & 1 \\
\hline & & & L & 2 & 2 & 4 \\
\hline \multirow[t]{2}{*}{5} & $\mathrm{~T} 2 \mathrm{~b}$ & 8 & $\mathrm{R}$ & 6 & 4 & 2 \\
\hline & & & L & 2 & 8 & 2 \\
\hline \multirow[t]{2}{*}{6} & $\mathrm{~T} 2 \mathrm{~b}$ & 9 & $\mathrm{R}$ & 1 & 4 & 1 \\
\hline & & & L & 1 & 3 & 3 \\
\hline \multirow[t]{2}{*}{7} & T2a & 7 & $\mathrm{R}$ & 3 & 4 & 3 \\
\hline & & & L & 2 & 2 & 0 \\
\hline \multirow[t]{2}{*}{8} & T3b & 9 & $\mathrm{R}$ & 3 & 2 & 5 \\
\hline & & & L & 1 & $2(1)$ & 0 \\
\hline \multirow[t]{2}{*}{9} & ТЗа & 7 & $\mathrm{R}$ & 1 & $3(1)$ & 1 \\
\hline & & & L & 3 & 3 & 0 \\
\hline \multirow[t]{2}{*}{10} & ТЗа & 7 & $\mathrm{R}$ & 1 & 2 & 0 \\
\hline & & & L & 2 & 2 & 1 \\
\hline \multirow[t]{2}{*}{11} & T3c & 7 & $R$ & 3 & 3 & 1 \\
\hline & & & L & 1 & 1 & 1 \\
\hline \multirow[t]{2}{*}{12} & T3c & 9 & $\mathrm{R}$ & 1 & 1 & 1 \\
\hline & & & L & 1 & 1 & 1 \\
\hline \multirow[t]{2}{*}{13} & $\mathrm{~T} 2 \mathrm{~b}$ & 6 & $\mathrm{R}$ & 1 & 1 & 1 \\
\hline & & & L & 2 & 5 & 1 \\
\hline \multirow[t]{2}{*}{14} & T2a & 7 & $\mathrm{R}$ & 1 & 1 & 1 \\
\hline & & & L & 1 & 2 & 1 \\
\hline
\end{tabular}

showed that adenocarcinoma had almost entirely infiltrated the left obturator $\mathrm{LN}$ and that most of the normal $\mathrm{LN}$ was replaced by the cancer cells (Fig. 2, part B).

In case $\# 9$, the serum PSA was $8.32 \mathrm{ng} / \mathrm{mL}$ and pathological examination showed LN metastasis in the right obturator LN that had not been found to be highly fluorescent. On the other hand, highly fluorescent LN was showed in the right internal iliac LN both in vivo and ex vivo, which does not have metastasis (Fig. 3, part A). The Gleason score from the surgical specimen was $3+4=7$. The bulk of the cancer expanded beyond the prostatic capsule at the left ventral side. Pathological examination of the prostate revealed adenocarcinoma with scattered lymphatic vessel invasion. Pathological examination showed that both normal LN structure and infiltration of adenocarcinoma coexisted in the right obturator LN (Fig. 3, part B).

The LN intensity under in vivo probing was 2.39 for external iliac LNs, 3.13 for obturator LNs and 8.84 for internal iliac LNs, and the intensity for the internal iliac 
Lymphatic pathway identified by fluorescence navigation

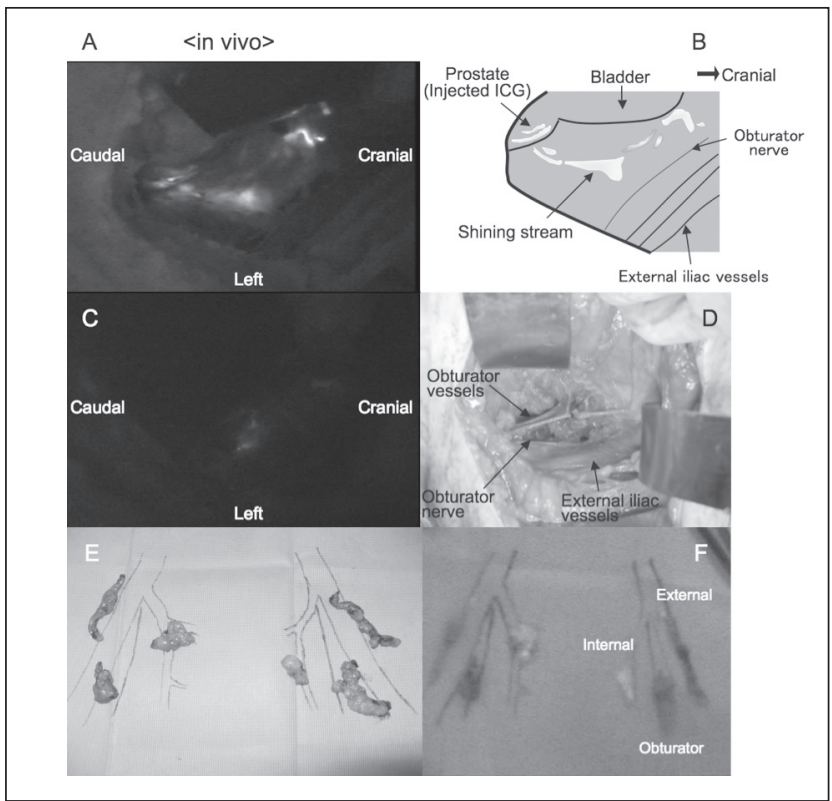

Fig. 1. Lymph node (LN) mapping by the fluorescence navigation system in case \#5 (Gleason score $=3+4$, negative LN involvement); A: In vivo probing of lymphatic channel around prostate before pelvic lymph node dissection (PLND) was shown. Note that high fluorescent of lymphatic flow was identified in the internal iliac region after direct injection of ICG; B: Schema of lymphatic vessels detected by the FN system was depicted; $\mathrm{C}$ : In vivo probing of lymphatic channel around prostate after PLND was shown. Note that high fluorescence compatible with lymphatic channel or LN was not overt after PLND with the aid of current FN system; D: Left side of the pelvic cavity was shown after PLND. Note that complete dissection of the pelvic LN was possible to be performed; E: Dissected LNs were placed and ordered along the pelvic vessels; F: Ex vivo probing of dissected pelvic LNs was shown. Note that high fluorescence was demonstrated in the internal iliac LN, but not in the external iliac or obturator LN.

nodes was significantly $(p<0.01)$ higher than the intensities for the other LNs (Fig. 4, part A). Ex vivo probing also demonstrated a high intensity for internal iliac nodes (9.91), lower intensities for obturator nodes (6.86) and external iliac nodes (5.49); the intensity for the internal iliac nodes was significantly $(p<0.01)$ higher than the intensities for the other LNs (Fig. 4, part B). The ratio of the intensity (in vivo) divided by number of LNs was significantly higher in internal iliac nodes than either the external iliac or obturator node $(p<0.01)$ (Fig. 4, part C).

\section{Discussion}

Recent publications have shown that SLN concept applies to cancers involving the skin and breast, ${ }^{1,2}$ but whether it applies to prostate cancer is debatable. Single photon emission computed tomography fused with computed tomography or magnetic resonance imaging has been shown to improve spatial resolution or orientation, thus allowing for a more precise localization of ${ }^{99 m}$ Tc-containing lymph node. ${ }^{8}$ The

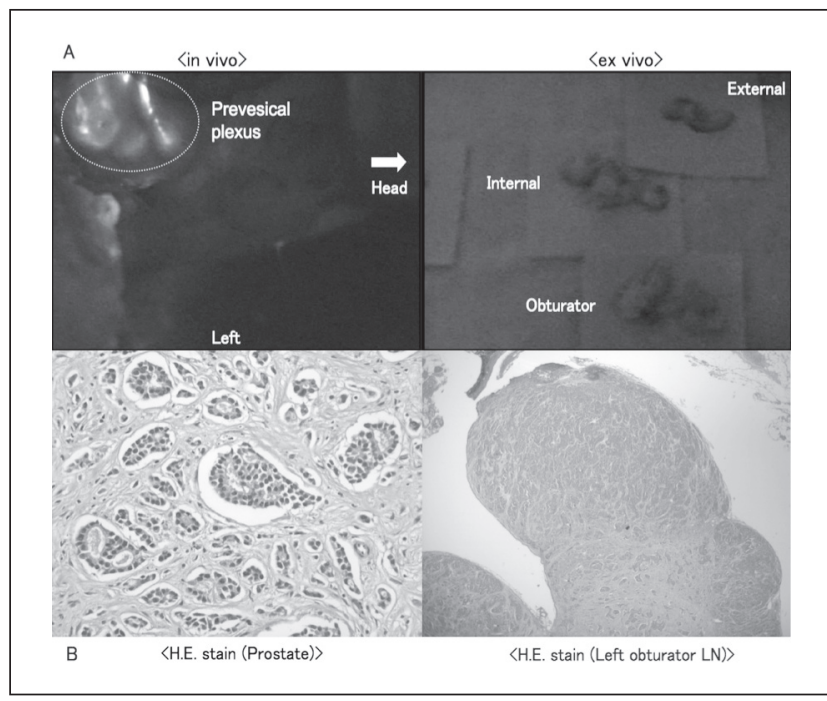

Fig. 2. Pathological lymph node (LN) involvement in relation to the lymphatic pathway determined by the fluorescence navigation system (Case \#8, left side); A: Though pathological examination showed metastasis in the left obturator $L N$, there was no strong fluorescence in the regional LNs of the prostate either in vivo or ex vivo. On the other hand, there was highly fluorescent region in the perivesical plexus, which was outside the region of extended LN dissection; B: Pathological examination revealed that adenocarcinoma with a Gleason score of 9 had diffusely invaded lymphatic vessels in the prostate and almost entirely infiltrated the left obturator LN

sensitivity of the radioguided SLN dissection for detecting patients with positive nodes is extremely high (96\%). ${ }^{9}$ This procedure, however, is time consuming and depends on the skills and endurance of the reader. Moreover, experience with this tool is limited, and it cannot overcome the problem of false-negative nodes. ${ }^{10}$ Because the conventional approach to determining $\mathrm{LN}$ involvement uses RI, which has several disadvantages owing to biohazardous potential, we have developed an easier and more accurate technique, in which ICG is injected into the prostate directly during RRP so that charge-coupled-device camera can be used to identify lymphatic channels and determine the location of the SLN.

Indocyanine green is a popular diagnostic reagent that is clinically approved for use in testing the liver function and measuring cardiac output. ${ }^{11}$ The incidence of adverse effects induced by ICG injection is $0.17 \%$, with an only $0.02 \%$ rate of shock-related symptoms. In our preliminary results, no adverse effects related to ICG injection were found, which was consistent with the previous reports. ${ }^{11}$

The primary focus of this study was to demonstrate the lymphatic channel that might be affected by the LN involvement of prostate cancer cells. Three lymphatic pathways have been proposed to be related to prostate cancer metastases, namely the ascending, lateral and posterior lymphatic channels. ${ }^{6}$ The clinical superiority of the FN system over the Rl-assisted approach is due to the ease of tracking the lymphatic vessels into the pelvic LNs under FN guid- 
Inoue et al.

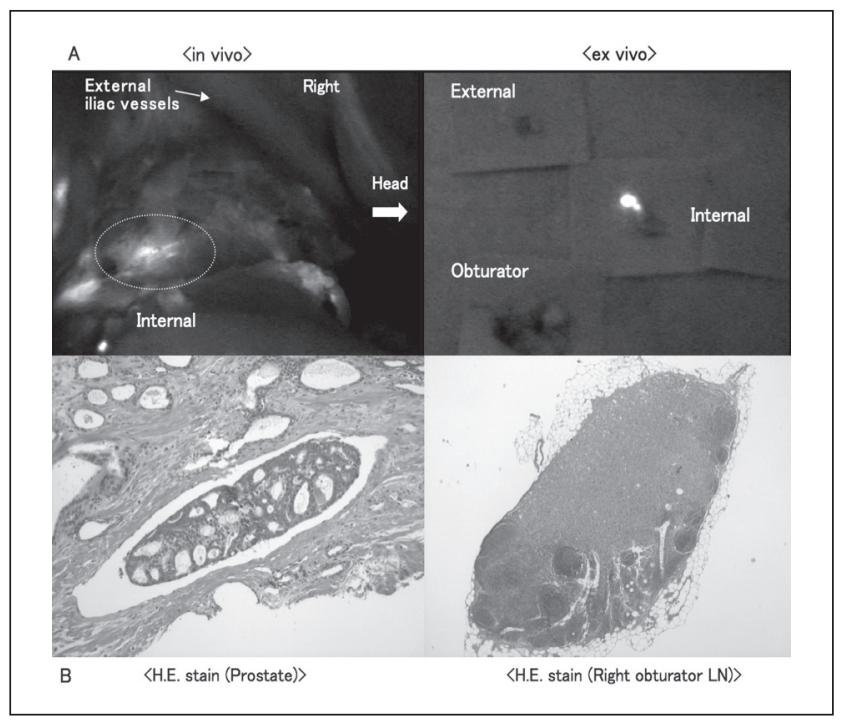

Fig. 3. Pathological lymph node (LN) involvement in relation to lymphatic pathway identified by the fluorescence navigation system (Case \#9, right side); A: Pathological examination showed that the right obturator LN had metastasis that had not showed up as a highly fluorescent region. On the other hand, a highly fluorescent region showed up in the right internal iliac LN, which did not have metastasis; B: Pathological examination revealed adenocarcinoma with a Gleason score of 7 scattered lymphatic vessel invasions in the prostate and that both normal LN structure and infiltration of adenocarcinoma coexisted in the right obturator LN.

ance and to the accuracy with which the FN system can identify the resected $L N$ as the SLN. The results we obtained with this FN approach showed that the intensity of ICG fluorescence in in vivo or ex vivo probing was the strongest in the internal iliac region, the next strongest in the obturator region, and the weakest in the external iliac region.

A well-understood anatomical approach to identifying the major and proper lymphatic pathway could help establish a more accurate direction of PLND. The clinical significance of PLND appears to be another issue, but has been discussed or emphasized in 3 different ways, such as the diagnostic nature of predicting prognosis, early recognition of additional therapy due to positive LN involvement, and possible therapeutic value of nodes dissection involved in the cancer cells. ${ }^{12-14}$ Given the present results, we think that the internal iliac region, not only obturator or external iliac nodes, should be the target of the PLND.

Our study has several significant limitations. First, the total case number was very small $(n=14)$. Second, SLN was not able to identify all metastatic LNs due to micrometastases blocking the lymphatic drainage of ICG into the LNs. Third, in our FN system, we were able to dissect out and obtain 155 LNs, only 2 of which were positive for cancer cells (cases \#8 and \#9). In case \# 8, it seemed that the primary lymphatic flow was obstructed by the infiltration of cancer cells and subsequent perivesical plexus was developed as a collateral pathway. The pathological review of these \#8 and \#9 cases validated the partial and/or complete obstruction of lymphatic pathway by infiltrating cancer cells, which might promote the development of alternative pathways. In turn, the potential drawback when identifying the SLN in prostate cancer appears to be closely associated with malignant potential of individual cancers. In the case of non-visualized LN rather than brightly visualized one under FN system, intra- and/or inter-communication of lymphatic network around prostate might accelerate the induction of alternative lymphatic pathway. Hence, intra-operative fluorescence is better suited to detect negative nodes than positive nodes and thus may not identify positive nodes. In the future, intraoperative evaluation of lymphatic channel by FN system could optimize and/or define a proper range of PLND in prostate cancer.

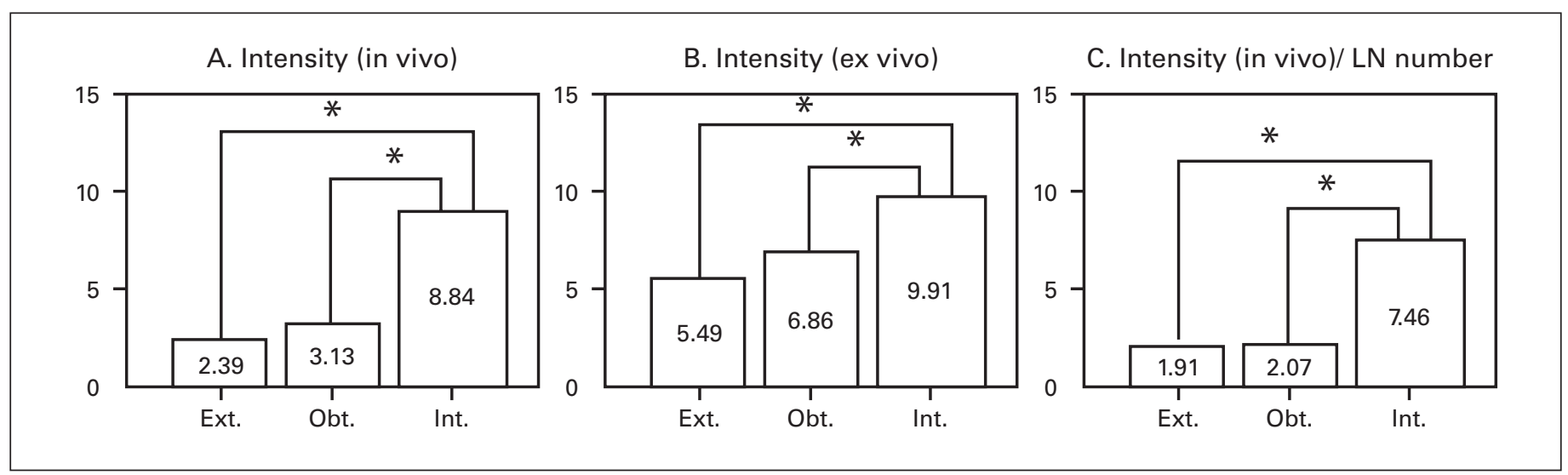

Fig. 4. Intensity of the indocyanine green fluorescence in lymph nodes (LNs) determined by the fluorescence navigation system was shown; A: In in vivo probing, the fluorescent intensity was higher in the internal iliac nodes than in the external iliac or obturator nodes ( $p<0.01$, each); B: In ex vivo probing, likewise, the fluorescent intensity of the internal iliac nodes was the highest compared with the external iliac or obturator nodes $(p<0.01$, each); C: The ratio of the intensity (in vivo probing) divided by the number of LNs, which represents the relative intensity of each LN, was significantly higher in the internal iliac nodes than external iliac or obturator nodes $(p<0.01)$. 
Lymphatic pathway identified by fluorescence navigation

\section{Conclusions}

The major lymphatic pathway involving the spread of prostate cancer appears to be the channel related to internal iliac LNs, which means that standard PLND covering the external iliac and obturator regions would not keep the cancer cells from spreading. Since the current results do not approve the SLN concept applicable to prostate cancer, further validation study to determine whether or not SLN biopsy guided by FN is essential in the treatment of prostate cancer is ongoing.

Competing interests: None declared.

This paper has been peer-reviewed.

\section{References}

1. Morton DL, Cochran AJ, Thompson JF, et al. Sentinel node biopsy for early-stage melanoma: accuracy and morbidity in MSLT-I, an international multicenter trial. Ann Surg 2005;242:302-11.

2. Posther KE, Wilke LG, Giuliano AE. Sentinel lymph node dissection and the current status of American trials on breast lymphatic mapping. Semin Oncol 2004;31:426-36.

3. Cabanas RM. An approach for the treatment of penile carcinoma. Cancer 1977; 39:456-66.
4. Wawroschek F, Vogt H, Weckermann D, et al. The sentinel lymph node concept in prostate cancer - first results of gamma probe-guided sentinel lymph node identification. Eur Urol 1999;36:595-600.

5. Jeschke $S$, Beri A, Grüll $M$, et al. Laparoscopic radioisotope-guided sentinel lymph node dissection in staging of prostate cancer. Eur Urol 2008:53:126-32.

6. Gil-Vernet JM. Prostate cancer: anatomical and surgical considerations. Br J Urol 1996;78:161-8.

7. Kitai $T$, Inomoto $T$, Miwa $M$, et al. Fluorescence navigation with indocyanine green for detecting sentinel lymph nodes in breast cancer. Breast Cancer 2005;12:211-5.

8. Mattei A, Fuechsel $\mathrm{FG}$, Bhatta Dhar $\mathrm{N}$, et al. The template of the primary lymphatic landing sites of the prostate should be revisited: results of a multimodality mapping study. Eur Urol 2008:53:118-25.

9. Weckermann D, Dorn R, Trefz M, et al. Sentinel lymph node dissection for prostate cancer: experience with more than 1,000 patients. J Urol 2007;177:916-20.

10. Briganti A, Blute ML, Eastham JH, et al. Pelvic lymph node dissection in prostate cancer. Eur Urol 2009:55:1251-65.

11. Kusano M, Tajima Y, Yamazaki K, et al. Sentinel node mapping guided by indocyanine green fluorescence imaging: a new method for sentinel node navigation surgery in gastrointestinal cancer. Dig Surg 2008;25:103-8.

12. Bader $P$, Burkhard $\mathrm{FC}$, Markwalder R, et al. Disease progression and survival of patients with positive lymph nodes after radical prostatectomy. Is there a chance of cure? I Urol 2003;169:849-54.

13. Allaf ME, Palapattu GS, Trock BJ, et al. Anatomical extent of lymph node dissection: impact on men with clinically localized prostate cancer. J Urol 2004;172:1840-4.

14. Daneshmand S, Quek ML, Stein JP, et al. Prognosis of patients with lymph node positive prostate cancer following radical prostatectomy: Iong-term results. J Urol 2004;172:2252-5.

Correspondence: Dr. Shogo Inoue, Department of Urology, Graduate School of Biomedical Sciences, Hiroshima University, 1-2-3 Kasumi, Minami-ku, Hiroshima 734-8551; fax: -81-82-257-5244; inosyogo@hiroshima-u.ac.jp 